\title{
Determination of $x$-ray elastic constants using an in situ pressing device
}

\author{
D. H. J. Teeuw and J. Th. M. De Hosson \\ Department of Applied Physics, Materials Science Centre, University of Groningen, \\ Nijenborgh 4, 9747 AG Groningen, The Netherlands
}

(Received 27 March 1997; accepted 7 November 1997)

The experimental determination of x-ray elastic constants are performed by in situ measurements of the dependence of the strain state in selected crystallites for different applied external compressive stresses. The use of compressive applied stresses instead of tensile applied stresses is of interest for $\mathrm{x}$-ray elastic constant determinations for materials which exhibit brittle crack-like behavior, which cannot be loaded to high tensile stresses in, for example, four-point bending devices. The x-ray elastic constants for $\{146\} \alpha-\mathrm{Al}_{2} \mathrm{O}_{3}$ are determined with the pressing device and compared to calculated as well as experimentally determined values which were tested in tensile loading devices.

X-ray residual stress measurements are based on the determination of elastic lattice strains in polycrystalline materials, which are to be converted to macroscopic stress states using linear elasticity theory. ${ }^{1}$ In this conversion, material elastic constants are required. For these material elastic constants often the macroscopic elastic constants, Young's modulus, and Poisson's ratio, are used. However, this procedure does not necessarily lead to accurate results for the determination of the absolute values of the residual stress state in the material, since the macroscopic elastic constants are an average of all the single-crystal elastic constants for different lattice planes in the material. ${ }^{2,3}$ For instance, if a specimen is textured, the macroscopic elastic constants do not correspond to the crystallographic plane specific $\mathrm{x}$-ray elastic constants. Consequently, it is not possible to determine absolute values for the residual stress state in the material. More accurate values of the absolute residual stress state are determined when the crystallographic plane specific X-ray elastic constants $1 / 2 s_{2}^{\{h k l\}}$ and $s_{1}^{\{h k l\}}$ are used instead. These x-ray elastic constants can be determined when a specimen is loaded in situ inside a diffractometer and the lattice spacing deviations are measured for different specimen orientations and applied loads.

Uniaxial tensile loading or four-point bending are usually employed in $\mathrm{x}$-ray elastic constant determination. ${ }^{4,5}$ In the determination of $\mathrm{x}$-ray elastic constants of materials which exhibit brittle crack-like behavior, these techniques run into problems because of mechanical failure of the specimen.

In compressive loading, in contrast to experiments which make use of four-point bending devices, there is no gradient in the applied stress as a function of depth from the surface. Especially for materials which have larger penetrations depths, like ceramics, the materials volume sampled by the $\mathrm{x}$-rays should contain a homogeneous stress state. With the pressing device, a homogeneous uniaxial stress state is applied throughout the sample.

Determining the $\mathrm{x}$-ray elastic constants from an applied compressive stress state should deliver equivalent values as determining the x-ray elastic constants from an applied tensile stress state, since the elastic response of an atom around its equilibrium position is equivalent for positive as well as negative displacements. The fracture toughness, however, is typically $\sim 10$ times larger in compression than for tensile loads. This is a consequence of the resistance of the material to plastic flow and the insensitivity of defects to compressive stresses. Because of the larger compressive strength, the specimen can withstand larger loads in compression; hence, a larger range of applied loads can be employed in the determination of the x-ray elastic constants.

Since the specimen is produced by sintering powder compacts, the distribution of crystallites in the specimen is random, ensuring the isotropic elastic properties of the specimen.

In x-ray diffraction, lattice spacings $d$ of $\{h k l\}$ lattice planes in crystallites are measured from the angular position $\theta$ of diffraction profiles through Bragg's law $n \lambda=2 d \sin \theta{ }^{6}$ The measured $d$-values correspond to the lattice plane set with orientation $\phi$ and $\psi$, as presented in Fig. 1. When a specimen is subjected to internal or applied stresses (IS and $A S$, respectively), the measured lattice spacing $d$ will deviate from the values measured for unstressed specimens. This deviation is normally expressed as the strain $\epsilon_{33}$ and described by $\epsilon_{33}=\left(d_{\phi, \psi-d_{0}}\right) / d_{0}$. Transformation of $\epsilon_{33}$ from the laboratory to the specimen system results in

$$
\begin{aligned}
\epsilon_{33, \phi=0, \psi}= & \frac{1}{2} s_{2}^{\{h k l\}}\left(\sigma_{11}^{I S}+\sigma^{A S}\right) \sin ^{2} \psi \\
& +s_{1}^{\{h k l\}}\left(\sigma_{11}^{I S}+\sigma_{22}^{I S}+\sigma^{A S}\right) .
\end{aligned}
$$




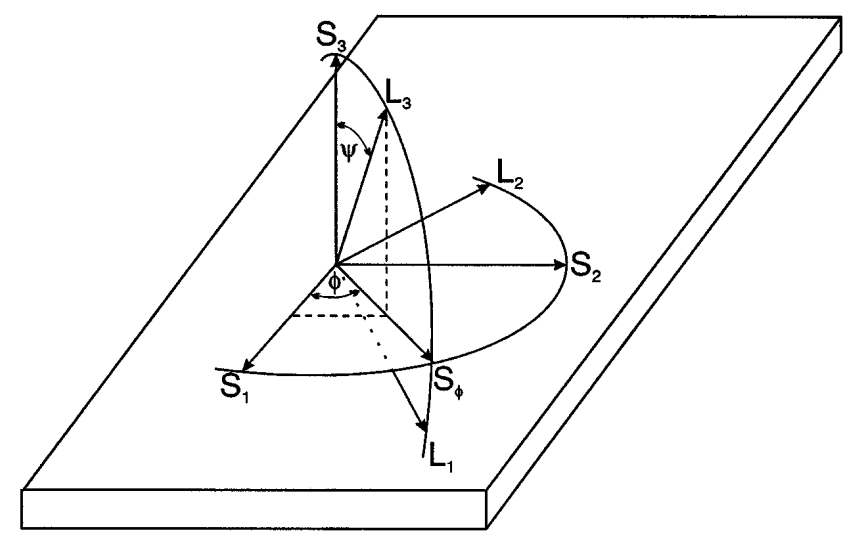

FIG. 1. Definition of the laboratory coordinate system $L_{i}$, sample coordinate system $S_{i}$, and the angles $\phi, \psi$.

Differentiation of this relation with respect to the applied stress and for the specific specimen orientation delivers readily the X-ray elastic constants $1 / 2 s_{2}^{\{h k l\}}$ and $s_{1}^{\{h k l\}}$ for the lattice planes of interest.

$$
\begin{aligned}
\frac{\delta^{2} \epsilon_{33, \varphi=0, \psi}}{\delta \sin ^{2} \psi \delta \sigma^{A S}} & =\frac{1}{2} s_{2}^{\{h k l\}} \\
\frac{\delta \epsilon_{33, \varphi=0, \psi=0}}{\delta \sigma^{A S}} & =s_{1}^{\{h k l\}} .
\end{aligned}
$$

If an external load is applied to a specimen of specific dimensions, the uniaxial stress exerted can be calculated from the dimensions of the specimen. The measured $d$-values for crystallites can be plotted against their orientation $\sin ^{2}(\psi)$. Doing so for several applied loads delivers a set of relations between measured $d$-values, crystallite orientations, and applied load. The curves that describe the relation between $d$-values and specimen orientation for different applied loads all intersect in one single point, $\left\{\sin ^{2}(\psi), d_{0}\right\}$, which gives the stress-free lattice spacing and orientation. Using the determined $d$-values and the stress-free lattice spacing $d_{0}$, the strains $\epsilon_{33, \phi=0, \psi}$ can be calculated. The derivatives of the lines plotted against the specimen orientation then deliver the $\mathrm{x}$-ray elastic constants.

The material of interest is $\alpha-\mathrm{Al}_{2} \mathrm{O}_{3}, 99.7 \%$ chemical purity. Dry pressing is used to mold the powder into beam-shaped green products which are sintered to high density. The specimens are texture-free and have straight and parallel edges. The lattice planes from which the lattice strains are determined are the $\{146\}$ planes, which have their Bragg diffraction angle at $136.30^{\circ} 2 \theta$ for $\mathrm{Cu} \mathrm{K}{ }_{\alpha}$ radiation. This reflection is chosen because of the high $2 \theta$ angle and the isolation of the reflection from other peaks, which makes it possible to perform background correction and $K \alpha_{2}$-stripping.

The x-ray elastic constant measurements are performed on a $\omega$-goniometer equipped with the in situ pressing device, which can deliver and register loads up to $10^{6} \mathrm{~N}$, using a piezo electric transducer. Such high loads may cause the pressing device to deform elastically, but this does not affect the actual measurements, since the load is measured in line with the specimen.

The specimens that are used need to be plan parallel. However, slight deviations are cancelled out by the self-aligning properties of the pressing device. The dimensions of the specimens used are maximum $10 \times$ $3 \times 3 \mathrm{~mm}$, whereas the width of the specimens must be $3.0 \mathrm{~mm}$. This is to ensure the compressive stress state in the specimen is uniaxial.

Since the nature of the experimental determination requires measurements to be compared, accurate control of the sample displacement from the optical axis of the diffractometer becomes an important issue. If the height of the sample is not correctly adjusted, an error in $2 \theta$ determination is made, resulting in erroneous values for the measured $d$-values. As a check on correct height adjustment, a layer of stress-free silicon powder of appropriate grain size is put onto the specimen. The $\{311\}$ silicon reflections are used as a check on displacement before and after the successive $d$-value determinations for the different applied loads.

The x-ray elastic constant measurements are carried out on a beam-shaped specimen with dimensions $9 \times$ $3 \times 3 \mathrm{~mm}$. Four different applied stresses are used, ranging from $-43 \mathrm{MPa}$ up to $-170 \mathrm{MPa}$. For five different specimen orientations, $\psi$, the corresponding Bragg-diffraction angle $2 \theta$ is determined. The $2 \theta$ range over which the profiles are measured is chosen in such a way that at both sides of the profile some background is measured. This makes it possible to subtract the background and apply $K \alpha_{2}$-stripping in order to determine the Bragg reflection as accurately as possible.

To check whether the stress state in the specimen is uniaxial, as assumed from the design of the pressing device, the diffraction angle $2 \theta$ should be determined as a function of $\psi$ and $\phi$, delivering the principal stresses and their direction. However, due to geometrical limitations in the design of the pressing device, $\phi$ tilting is not allowed.

With the correctly aligned specimen, the lattice strains as a function of applied stress and specimen orientation are determined. The measured $d$-values are depicted in Fig. 2. From this graph, the strain-free lattice spacing $d_{0}$ can be determined as the intersection point of the four lines. The correct value for $d_{0}$ can be found using a method proposed in Ref. 7. The intercept at $\sin ^{2} \psi=0$ of the tangent to the $d_{\phi=0, \psi}^{\{h k l\}}$ versus $\sin ^{2} \psi$ curves is plotted versus the corresponding slope, as is depicted in Fig. 3. For the straight line through these data points, it holds that

$$
d_{\phi=0, \psi}^{\{h k l\}}=d_{0}-\sin ^{2} \psi_{0} .
$$




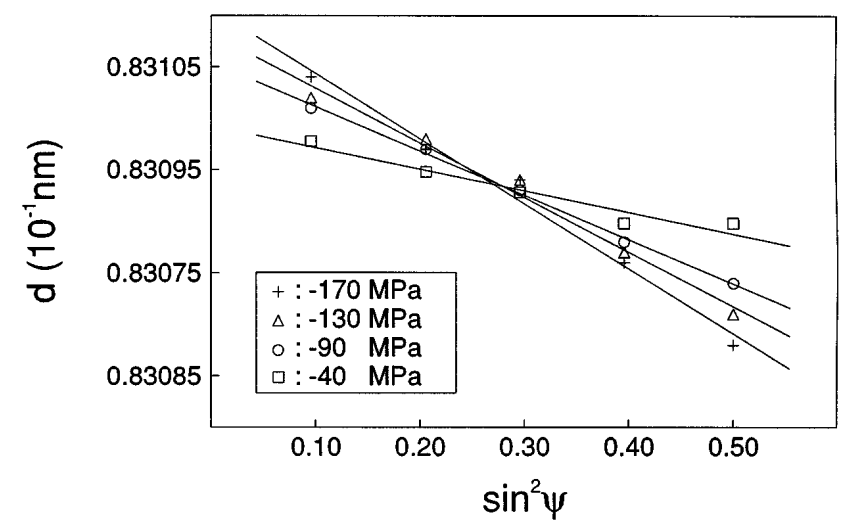

FIG. 2. $d$-values as a function of specimen orientation for different applied loads.

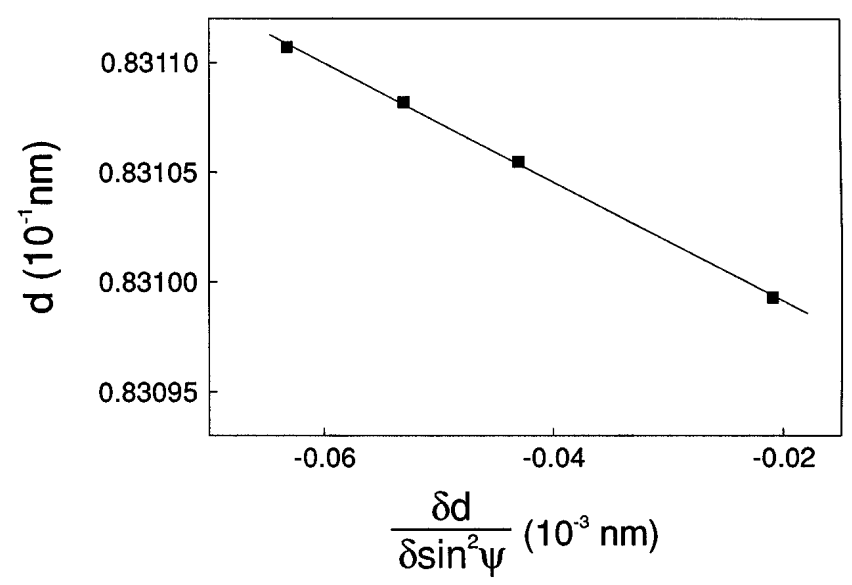

FIG. 3. Determination of the intersection point.

From the slope and intercept of this line, values for $\sin ^{2} \psi_{0}$ and $d_{0}$ can be found; see Table I.

With the strain-free lattice spacing, the measured $d$-values are then converted to the lattice strains $\epsilon_{33, \phi=0, \psi}$. Plotting the measured strains against the applied stresses for the different specimen orientations, Fig. 4, delivers the relation described in Eq. (2).

The slopes of the curves depicted in Fig. 4 are plotted against the specimen orientation, as displayed in Fig. 5. The intersection of this line with the strain axis delivers the $s_{1}^{\{h k l\}}$ value. The slope of the line represents the $1 / 2 s_{2}^{\{h k l\}}$ value. Both values are summarized in Table II together with the averaged values calculated according to Voigt and Reuss as well as values determined in four-point bending and tensile loading experiments. ${ }^{2,8}$

Using the derived value for $1 / 2 s_{2}^{\{h k l\}}$, the stress state in the specimen is derived from the slopes in Fig. 2. Correcting the derived values for the applied stress delivers an average value for the residual stress state in the specimen, $\sigma^{I S}=57 \pm 3 \mathrm{MPa}$.

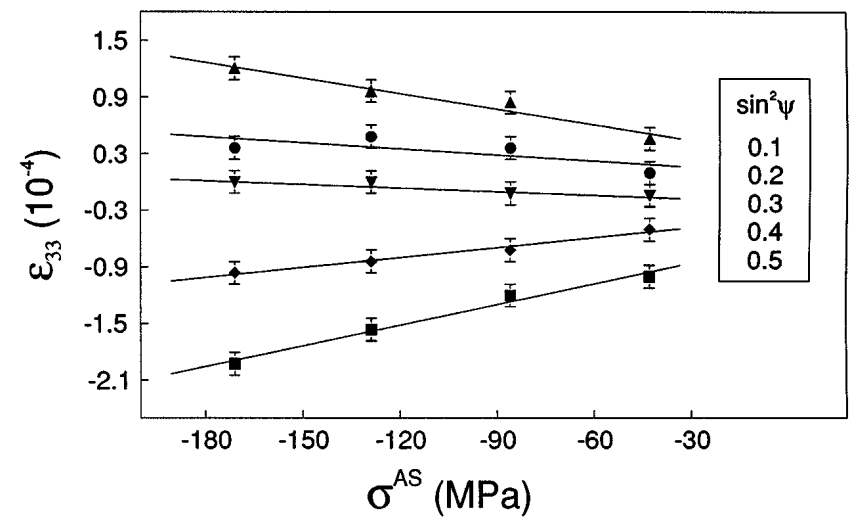

FIG. 4. Lattice strains as a function of applied stress for different crystallite orientations.

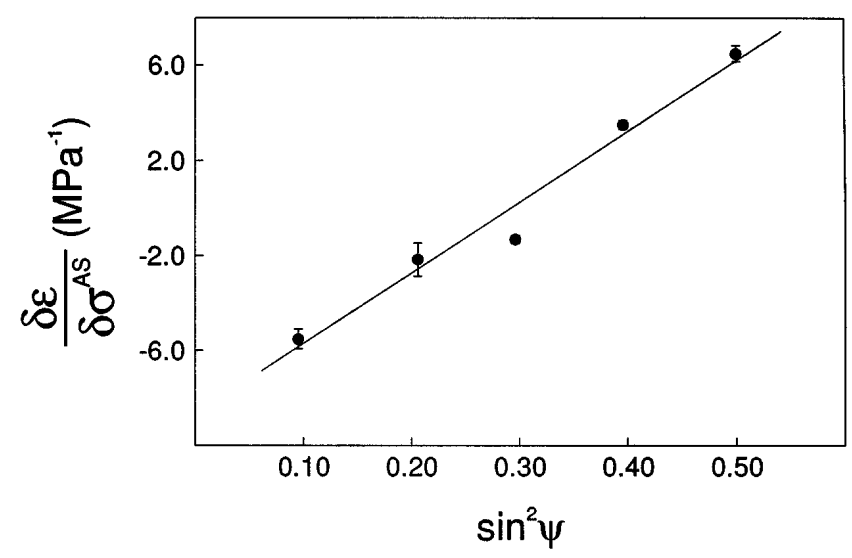

FIG. 5. X-ray elastic constants determination.

TABLE I. Stress-free lattice spacing.

\begin{tabular}{ll}
\hline \hline$d_{0}(\mathrm{~nm})$ & $0.0830935 \pm 0.0000004$ \\
$\sin ^{2} \psi_{0}$ & $0.272 \pm 0.006$ \\
\hline \hline
\end{tabular}

TABLE II. X-ray elastic constants.

\begin{tabular}{lcc}
\hline \hline \multicolumn{1}{c}{ Method } & $S_{1}\left(* 10^{-6} \mathrm{MPa}^{-1}\right)$ & $1 / 2 s_{2}\left(* 10^{-6} \mathrm{MPa}^{-1}\right)$ \\
\hline Measured here & $-0.83 \pm 0.02$ & $2.97 \pm 0.07$ \\
Calculated & -0.76 & 3.75 \\
Four-point bending & -0.42 & 3.06 \\
& $-0.92 \pm 0.15$ & $3.36 \pm 0.12$ \\
Tensile loading & $-0.56 \pm 0.02$ & $3.08 \pm 0.14$ \\
\hline \hline
\end{tabular}

From Table II a large difference is observed among the various experimental configurations. In addition, neither of the values calculated by the Voigt or Reuss hypotheses correspond to the values measured. These calculations, however, assume the material to be texture free, with a theoretical density and without second phases involved.

From the literature it is known that microstructural properties of the material under investigation may have 
a significant influence on the determined $\mathrm{x}$-ray elastic constants. ${ }^{8}$ The determined values for the $\mathrm{x}$-ray elastic constants depend, for instance, on the porosity of the specimen under investigation, and also the presence of a second phase influences the values measured. The materials used in this study were $99.7 \%$ pure and sintered to $99 \%$ density. Therefore, it is expected that the determined x-ray elastic constants deviate from the constants for pure, theoretically dense alumina. However, considering the relatively small error in the values determined, residual stress measurements on this type of alumina can be accurately performed using the $\mathrm{x}$-ray elastic constants determined.

The determination of the x-ray elastic constants for brittle materials can be performed accurately with the in situ pressing device described In contrast to measurements which are performed under tensile loading, the compressive loads that can be applied to the specimen may reach high values before mechanical failure of the specimen.
The residual stress state in the material, as determined from the loading curves using the derived x-ray elastic constant, delivered comparable results for the different applied loads, indicating that the x-ray elastic constants determined hold for this type of alumina.

\section{REFERENCES}

1. I. C. Noyan and J. B. Cohen, Residual Stress (Springer-Verlag, New York, 1987).

2. B. Eigenmann and E. Macherauch, Mat.-wiss. u. Werkstofftech. 26, 199-216 (1995)

3. J. A. Sue, Surf. Coat. Technol. 54/55, 154-159 (1992).

4. M. Kurita, Nondestr. Test. Eval. 8-9, 681-697 (1992).

5. D. Amos, B. Eigenmann, and E. Macherauch, Z. Metallkd. 5, 317-1323 (1994).

6. B.D. Cullity, Elements of X-ray Diffraction (Addison-Wesley, Reading, MA, 1978).

7. W. G. Sloof, B. J. Kooi, R. Delhez, Th.H. de Keijser, and E. J. Mittemeijer, J. Mater. Res. 6, 1440-1457 (1996)

8. K. Tanaka and Y. Yamamoto, ICRS2, edited by G. Beck et al. (Elsevier Applied Science, New York, 1989), pp. 328-334. 